\title{
Estrategias y modelos de negocio: aclaración de conceptos y terminología de la prensa en Internet
}

\author{
Manuel Goyanes MaRTíneZ \\ Universidad de Santiago de Compostela \\ Universidad de Westminster \\ goyanes_martinez@my.westminster.ac.uk
}

Recibido: $12 / 03 / 2012$

Aceptado: 30/10/2012

\section{Resumen}

Las estrategias y modelos de negocio de la prensa digital han sido objeto de estudio de una buena parte de académicos de la gestión mediática. El uso de terminología económica y del management ha provocado su utilización indiscriminada y cierta confusión entre ambos conceptos. La presente investigación contribuye a explicar y asentar términos sobre las estrategias y modelos de negocio desarrollados por la prensa digital. El objetivo es clarificar los términos y analizar los ejemplos de los diarios de referencia que los desarrollan.

Palabras clave: modelo de negocio, estrategia, cibermedios, terminología

\section{Strategies and Business Models: Clarification of Concepts and Terminology in the Digital Press}

\begin{abstract}
The strategies and business models of the digital press have been studied for a large part of media management scholars. The use of economic and management terminology has caused their indiscriminate utilization and some confusion between the concepts. This research contributes to explain and settle terms about the strategies and business models developed by the digital press. The goal is to clarify the concepts and examine the most important digital newspapers examples.
\end{abstract}

Keywords: business model, strategy, digital press, terminology.

\section{Referencia normalizada}

GOYANES MARTÍNEZ, Manuel (2013): "Estrategias y modelos de negocio: aclaración de conceptos y terminología de la prensa en Internet". Estudios sobre el mensaje periodístico. Vol. 19, Núm. 1, págs.: 419-431. Madrid, Servicio de Publicaciones de la Universidad Complutense.

Sumario: 1. Introducción y Objetivos. 2. Estrategia gratuita, modelo de negocio gratuito. 3. Estrategia propia de pago, modelos mixtos: fremium y metered model. 4. Estrategia propia de pago, modelo de negocio cerrado o paywall. 5. Alianzas estratégicas propias: limitadas y conjuntas. 6. Alianzas estratégicas ajenas. 7. Conclusiones. 8. Referencias bibliográficas.

\section{Introducción y objetivos}

La prensa gravitó su obtención de ingresos en la doble financiación (Two-side market, Rochet y Tirole, 2003) que implica tanto la venta del producto al lector como el recurso de publicidad (Sonnac, 2009). Sin lectores no hay anunciantes, y sin estos no hay ingresos. Internet alteró tanto las prácticas de los consumidores como la de los anunciantes, provocando cambios en estas dos fuentes de ingresos (Casero-Ripollés, 2010).

La publicidad es la primera fuente de ingresos en la mayoría de ediciones online (Mensing y Rejfek, 2005; Peng et al., 1999), y se espera mayoritariamente que continúe (Chyi y Sylvie, 2000; Bell, 2005). A pesar de que la publicidad en Internet au- 
menta consecutivamente cada año no logra compensar las pérdidas de las versiones impresas (Pfanner, 2007). El promedio de márgenes de beneficios de los cibermedios logra escasamente la mitad de lo alcanzado por el sector impreso (Huang, 2007).

Las compañías editoras comienzan a presionar a sus ediciones digitales a ganar dinero (Krüger et al., 2003). No obstante son muy pocos cibermedios los que han resultado exitosos imponiendo un modelo de ingresos cuyo sistema sea el pago del lector. Aunque en Estados Unidos (exceptuando algún caso como The Wall Street Journal y The New York Times y otros) el acceso es ampliamente de carácter gratuito (Strupp, 2007), muchos cibermedios británicos ya cobran por algún contenido (Herbert y Thurman, 2007).

Existe consenso entre los académicos en que la mayoría de cibermedios todavía no encontraron una estrategia empresaria y un modelo de negocio con los que se sientan ampliamente cómodos. Aunque muchos medios digitales han intentado nuevos enfoques (Crosbie, 2004), ninguno de los métodos ha generado una avalancha de imitadores.

Los medios de información digital vacilan sobre la adopción de modelos de ingresos distintos al tradicional por publicidad (Sylvie, 2008). La cuestión es descubrir los productos online por los que los consumidores estén dispuestos a pagar. La tesis más extendida para la implantación de estrategias de pago es que la información ofrecida debe crear valor, es decir, proporcionar una información diferenciada y no disponible en otros sitios de carácter gratuito.

Las estrategias y modelos de negocio de la prensa digital han sido objeto de estudio de una buena parte de académicos de la gestión mediática. El uso de terminología económica y del management ha provocado su utilización indiscriminada y cierta confusión entre conceptos. La presente investigación contribuye a explicar y asentar términos sobre las estrategias y modelos de negocio desarrollados por la prensa digital. El objetivo es clarificar los conceptos y términos y analizar los ejemplos de los diarios de referencia que los desarrollan.

Consideramos que el modelo de negocio no es lo mismo que la estrategia (Shafer et al., 2005; Casadesus y Ricart, 2009) pero muchos autores, como señala Magretta, (2002) utilizan el término "estrategia" y "modelo de negocio" de modo intercambiable. Aunque ambos términos se enfrentan a los mismos objetivos y problemas, ser rentables, consideramos que se deben dividir en niveles diferenciados (véase Casedesus y Ricart, 2009).

La literatura escrita alrededor de los cibermedios no iba ser excepción de modo que algunos autores confunden ambos términos, y hablan de modelos de negocio cuando en realidad se refieren al plano estratégico o viceversa. Nuestra visión de la estrategia se engloba dentro de un plan organizado, un patrón (Mintzberg, 1994), o una posición global (Drucker, 1980) a lo largo del tiempo (Jhonson, 2001). Una distinción práctica describe al modelo de negocio como un sistema que muestra como las piezas de un negocio encajan entre sí, mientras la estrategia incluye, la más de las veces, la competencia (Magretta, 2002). En general la mayoría de autores describe el modelo de negocio como una suma de elementos y relaciones que indican cómo una firma crea valor. 
Bajo esta perspectiva, dividimos las estrategias de la prensa digital en estrategias propias, o en alianzas estratégicas. Las estrategias propias pueden ser catalogadas como: gratuitas o de pago. Las estrategias propias gratuitas son las más abundantes (Strupp, 2007, Casero-Ripollés, 2010), mientras las estrategias de pago pueden adoptar diferentes modalidades. Las alianzas estratégicas (acuerdos empresariales) no obstante, son la mayoría de las veces de pago y las clasificamos asimismo como propias-limitadas (una plataforma y un creador, ej. Orbyt) o propias-conjuntas (una plataforma varios creadores, ej. Ongo). Por último las alianzas estratégicas ajenas son acuerdos empresariales entre un creador de contenido y varios grupos de comunicación (ej. Subscriciones de Apple)

A lo largo de los siguientes apartados explicaremos cada una de estas estrategias y modelos de negocio, centrándonos asimismo en los sistemas de ingresos desarrollados.

\section{Estrategia gratuita, modelo de negocio gratuito}

La prensa en formato papel fue tradicionalmente un ejemplo de rentabilidad (Picard, 1998, 1999). Los ingresos procedentes de la publicidad y la venta superaban ampliamente a los costes de producción, almacenamiento y distribución de los diarios (Varela, 2011; Shapiro y Variant, 1998; Larrañaga, 2010). De este modo las compañías periodísticas capturaban buena parte del valor creado en la producción de sus productos. En el terreno digital la cosa cambia de tal manera que en la mayor parte de casos los cibermedios no podrían operar como estructuras independientes (Nesbitt y Lavine, 2004; Ernst, 2007; Cohen, 2007). Además la mayoría de cibermedios optan por un modelo de negocio gratuito y financiamiento mediante publicidad (Mensing y Rejfek, 2005; Peng et al., 1999; Strupp; 2007).

Los beneficios de las ediciones digitales, generalmente $99 \%$ a través de publicidad, superan a los costes de producción del producto (nóminas, servidores, equipos, etc.) pero el volcado informativo procedente de las ediciones en papel es muy significativo en la mayoría de cibermedios. De esta forma es inevitable que hablemos de cibermedios como estructuras independientes.

En la prensa tradicional los ingresos por publicidad (IP) y la venta a lectores (VL) superan los costes incurridos en la producción intelectual (PI), la sustentación del producto en un formato (SP), el almacenamiento (A) y la distribución (D). De este modo podemos concluir que la prensa tradicional captura el valor suficiente para operar y para obtener beneficios: $\mathrm{IP}+\mathrm{VL}>\mathrm{PI}+\mathrm{SP}+\mathrm{A}+\mathrm{D}$.

Las ediciones digitales a priori capturan el suficiente valor como para ser rentables de tal manera que: la sustentación (S) y la logística que implica (L) (ambos costes) son menores que los ingresos publicitarios (IP). $\mathrm{S}+\mathrm{L}<\mathrm{IP}$, la empresa genera valor y lo

Generación de valor de la prensa tradicional: $\mathbf{I P}+\mathbf{V L}>\mathbf{P I}+\mathbf{S P}+\mathbf{A}+\mathbf{D}$

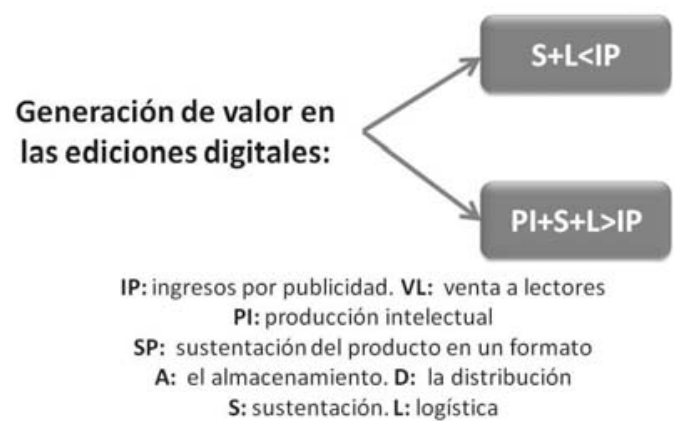


captura. Pero si tenemos en cuenta que la producción intelectual (PI) debería ser propia y no procedente de la edición tradicional, la cosa cambia: $\mathrm{PI}+\mathrm{S}+\mathrm{L}>\mathrm{IP}$, y por lo tanto ahora generan valor pero no lo capturan.

Los cibermedios con modelo de negocio gratuito únicamente son rentables como estructura independiente si $\mathrm{PI}+\mathrm{S}+\mathrm{L}>\mathrm{IP}$ pero no si $\mathrm{S}+\mathrm{L}<\mathrm{IP}$. De este modo, ¿Por qué la producción intelectual, la sustentación en un formato y la logística que implica no son mayores que los ingresos en la prensa digital, cuando desaparecen, incluso, dos eslabones de la cadena de valor de la prensa tradicional?

Resulta al menos curioso como en un ecosistema informativo como es el digital, a priori, con mayores facilidades, las compañías no son rentables en base a un modelo de negocio gratuito y financiamiento a través de publicidad. Las causas se encuentran principalmente en la disposición a no pagar por una commodity (KPMG, 2010; Pew Research, 2011; Picard, 2009) conjuntamente a la expansión de una profunda cultura de la gratuidad en Internet (Castells, 2000, 2009; Lessard, 2006).

Entonces si la disposición a pagar (DP) por un producto informativo en Internet es igual a cero, $\mathrm{DP}=0$, la captura de valor $(\mathrm{CV})$ únicamente procede de los ingresos publicitarios en base al modelo de negocio gratuito y descontando la sustentación (S) y la logística (L), CV=IP-(S+L). Lo que sucede es que el valor creado en las ediciones en papel a través de su producción intelectual no crea valor en las ediciones digitales con modelo de negocio gratuito puesto quien captura ese valor son los consumidores y no la empresa.

Que el usuario no esté dispuesto a pagar por un producto informativo que una compañía ofrece deliberadamente de forma gratuita no significa que el consumidor no reconozca que ese bien tiene un valor monetario intrínseco (VM). Lo que sucede es que ese valor monetario lo captura el consumidor ya que no lo paga, es decir, se produce un excedente del consumidor (EC). De este modo la disposición a pagar (DP) de un usuario por un producto informativo es igual a cero pero el valor monetario intrínseco al bien y que el usuario reconoce es igual a $\mathrm{X}$ que así mismo es igual al excedente del consumidor. Entonces: $\mathrm{DP}=\mathrm{X}=\mathrm{EC}$.

Los cibermedios con modelo de negocio gratuito se sustentan mediante ingresos publicitarios (Casero-Ripollés, 2010; Gallardo, 2011; Campos, 2010). Los ingresos publicitarios dependen de la audiencia del medio, puesto que en su variabilidad radican sus ingresos (Miguel de Bustos y Casado del Río, 2010; Miguel de Bustos, 2000). Mayor audiencia que la competencia significan mayores ingresos, por el contrario menos audiencia que la competencia significan menores ingresos. La compañía con mayor audiencia será, consecuentemente, la que más valor capture.

Dado que el elemento clave para obtener mayores ingresos en el modelo gratuito es la audiencia, los cibermedios intentarán tener más audiencia que sus competidores. Para tener mayor audiencia (crear valor) los cibermedios (tanto de pago como gratuitos) utilizan tres estrategias:

- Ofrecer una información que el usuario valora por algún motivo: exclusividad (Galiford, 2010), alto valor añadido (periodistas de prestigio) (Filloux, 2011), noticias de nicho y especializadas, personalización e individualización (Rodríguez-Martínez et al., 2010; Micó-Sanz y González-Molina, 2010) o la imposi- 
bilidad de búsqueda en otros sitios web de carácter gratuito (Chyi y Sylvie, 2001; Crosbie, 2004, Miguel de Bustos y Casado del Río, 2010).

- Aprovechar el valor de marca de las ediciones impresas: volcado mayoritario de las ediciones impresas en el ámbito digital. El cibermedio se aprovecha de la producción intelectual y del valor de marca (sobre todo credibilidad) del diario en papel (Crosbie, 2004, Albornoz, 2003, 2005; Millán, 2001).

- Combinar las dos anteriores: noticias que el usuario valore por algún motivo aprovechando el valor de marca de las ediciones impresas.

Muchas voces del mercado reclaman, además, una migración en bloque cara métodos de pago (Rojo-Villada, 2008) que implicaría la pérdida de competencia entre cibermedios gratuitos y de pago. Esta estrategia se está perfilando, en parte, a través de acuerdos empresariales que permiten la creación de nuevas plataformas como Orbyt o Kiosko y Más.

La estrategia más empleada por los cibermedios con modelos de negocio gratuitos es la de aprovechar el valor de marca de las ediciones impresas, volcando la mayor parte de las noticias y reciclando su producción intelectual. Al desaparecer el ingreso del lector, la información en los cibermedios está orientada a la generación de tráfico, que es el recurso clave (valor) percibido por los anunciantes para publicarse. Como la credibilidad y el valor de marca del que gozan los sitios web de las editoras de reconocidas cabeceras de prensa es un patrimonio ganado en el terreno offline (Salverría, 2005; Millán, 2001; Albornoz, 2003, 2005) resulta sencillo alcanzar amplias audiencias con informaciones de escaso valor para el lector, puesto que este no la paga, sólo la consume. Además autores como Picard (2009) ya comienza a hablar de las noticias como una commodity.

Como observamos el lector queda al margen de la disposición a pagar por la información que los cibermedios gratuitos ofrecen. En cambio los anunciantes sí están dispuestos a pagar por el tráfico que los cibermedios generan, capturando entonces una parte del valor que crean.

\section{Estrategia propia de pago, modelos mixtos: fremium y metered model}

El debate y discusión alrededor del pago por contenidos fue extenso desde los inicios de Internet. Una primera aproximación a la literatura muestra la existencia de dos corrientes con sendos partidarios y detractores.

Fueron muchos investigadores los que estudiaron el pago por información desde una perspectiva puramente económica (Schmalensee, 1984; Salinger, 1995; Varian, 1995, 2003; Fay y MacKie-Mason, 2001). Concretamente este fenómeno está ampliamente descrito en la literatura de la agrupación de los bienes informativos (bundling) donde ya existen varios modelos, explicando por qué su agrupación conduce a precios e ingresos más elevados en comparación con la desagregación (unbundled) en artículos individuales (Bakos y Brynsjolfsson, 1996; Fay y Mackie-Mason, 2001; Varian, 2001). No obstante no es siempre así (Bakos y Brynjolfsson, 1996).

Por otra parte el debate entre partidarios del pago por contenidos y la gratuidad fue estudiado desde una perspectiva económica pero también mediática, focalizando 
el análisis de las estrategias y en la comprensión de los modelos de negocio. La controversia establecida provocó el establecimiento de dos posturas ideológicas irreconciliables, que fue, si cabe, más vehemente en los partidarios de la gratuidad (Eisenberg, 2002; Zerdick et al. 1999).

No obstante, la discusión ideológica sobre el pago por contenidos tiene también un trasfondo económico, puesto que la venta de contenido online difiere de la venta offline. En este entorno los diarios y revistas online tienden cada vez más a ofrecer productos agrupados (bundled) y bajo pago.

La migración desde modelos de negocio gratuitos con sistemas o modelos de financiamiento mediante publicidad hacia modelos de ingresos basados en la subscripción o pago por uso tampoco resuelve los problemas de canibalización. En buena medida es así porque el descenso de los ingresos publicitarios es mayor que el aumento de los ingresos a través del pago por contenidos.

No obstante, la crisis del mercado publicitario digital ha forzado a los diarios online a la búsqueda de nuevas fuentes de ingresos. Una de ellas consiste en la oferta de contenido de pago como contenido premium por un lado, y contenido gratuito y financiamiento publicitario por otro. Se trata de una estrategia de pago con un modelo de negocio mixto a partir de un modelo o sistema de ingresos basado en publicidad,

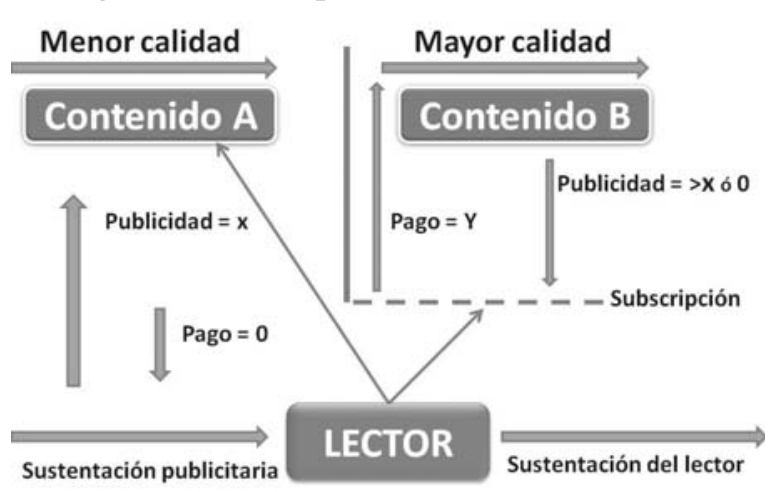

Representación del modelo de negocio fremium en la prensa digital. Elaboración propia. subscripciones y/o pago por uso. El modelo de negocio permite el establecimiento de dos contenedores informativos diferenciados: uno de máxima calidad de pago cuyo sistema de ingresos son las subscripciones o pago de los lectores, y otro de carácter funcional y operativo sustentado ampliamente por publicidad.

La táctica empleada en los contenidos de pago del modelo de fremium variará dependiendo de la cabecera. Las tácticas más empleadas son: $\mathrm{u}$ ofrecer el contenido de pago sin publicidad, o con publicidad.

El ejemplo más citado del modelo fremium es el del Wall Street Journal de Nueva York. El diario ofrece dos tipos de subscripción a los contenidos premium: por un lado una subscripción digital semanal de $£ 2.56$ con permanencia obligada de un año. La subscripción permite la consulta del diario en la web, el Smartphone o la tableta. La segunda oferta consiste también en una subscripción semanal digital más una impresa de £3.19 con permanencia obligada de un año (The Wall Street Journal, 2012).

The Wall Street Journal Digital Network (compañía que integra todas las plataformas digitales del grupo: WSJ.com; MarketWatch.com; Barrons.com; SmartMoney.com; AllThingsD.com y FINS.com.) es la empresa líder en oferta de contenido digital premium con más de 1.3 millones de subscriptores y una media de más de 50 millones de visitas únicas mensuales. The Wall Stree Journal digital ofrece una com- 
binación entre contenido gratuito y bajo subscripción con una audiencia total de más de 36 millones de visitas mensuales (The Wall Street Journal Digital Network, 2012).

El modelo metered model comienza a atraer a los grandes diarios que apuestan por el pago por contenidos y la combinación como modelo de ingresos (O'Regan, 2001; Filloux, 2011, EditorsWeblog, 2011). El modelo permite el acceso gratuito a un número limitado de artículos durante un tiempo concertado, para luego cobrar una vez que se exceda.

La principal característica es que la cabecera "mantiene" los usuarios únicos mensuales y sus ingresos mediante publicidad. Únicamente se penalizan los lectores "duros". La dificultad de implementación do modelo radica en la decisión táctica de cuantas noticias serán gratuitas y el precio de las mismas una vez que el usuario se subscriba. De este modo el modelo plantea diversos escenarios.

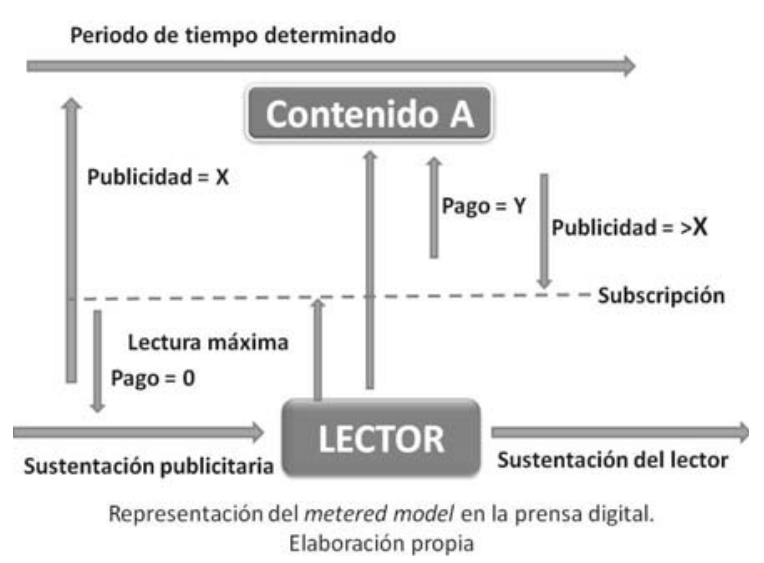

El precursor del metered model fue el Financial Times en el año 2007. Actualmente cuenta con más de 230.000 subscriptores y 3,7 millones de usuarios registrados (PaidContent, 2011a). El diario permite leer durante un mes un total de 8 artículos gratuitos previo registro en el cibermedio. Una vez superados, el lector debe subscribirse a una de las 5 ofertas.

La relativa rentabilidad del $\mathrm{Fi}$ nancial Times ha provocado la implementación de una fórmula semejante en el New York Times. El coqueteo del diario neoyorkino con las estrategias de pago no son nuevas. En el 2005 el diario ya había decidido la implementación del pago para las columnas del diario digital (TimesSelect) para finalizarlo a inicios del 2007. El diario cuenta con un total de más de 320.000 abonados (Q4) (PaidContent, 2011b) y unos ingresos de \$646 millones (Q4, NYT Co.) (PaidContent, 2011c), un $2.8 \%$ menos respecto al año anterior.

\section{Estrategia propia de pago, modelo de negocio cerrado o paywall}

El modelo de negocio cerrado o paywall, cosiste en la imposición de una barrera de pago entre el usuario y el contenido informativo. El usuario debe pagar para acceder al diario. Si no lo hace no accede. Se trata de la opción más arriesgada, puesto que la barrera provoca el descenso brusco del número de visitas únicas mensuales y consecuentemente de los ingresos mediante publicidad. El sistema de ingresos del paywall se basa fundamentalmente en las subscripciones (semanales, mensuales, anuales, etc.) pero también en el pago por uso.

La implementación del modelo de negocio cerrado de un cibermedio procedente de un modelo de negocio gratuito implica el siguiente escenario. Bajada brusca de las visitas únicas mensuales correlativamente al descenso mediante publicidad (Véase el 
ejemplo de The Times o New York Times, The Guardian, 2010; ComScore, 2010). Una vez implementado el modelo la compañía puede experimentar un ascenso consecutivo de subscripciones correlativamente a los ingresos publicitarios (personalizados a la audiencia). Por el contrario si la firma no consigue una ventaja competitiva y los lectores no valoran el contenido, los ingresos son menores que el anterior modelo gratuito, obligando a la firma a la retoma del modelo o al planteamiento de una nueva estrategia (Véase El País o New York Times con TimesSelct).

\section{Alianzas estratégicas propias: limitadas y conjuntas}

Las alianzas estratégicas propias las dividimos en limitadas o conjuntas. Ambas son acuerdos empresariales entre diferentes medios de comunicación digitales con los que migrar conjuntamente al pago, limitar la competencia gratuita y la canibalización. Asimismo ambas operan a través de un modelo de negocio cerrado con un sistema de ingresos basado en las subscripciones o en el pago por uso.

La principal diferencia radica

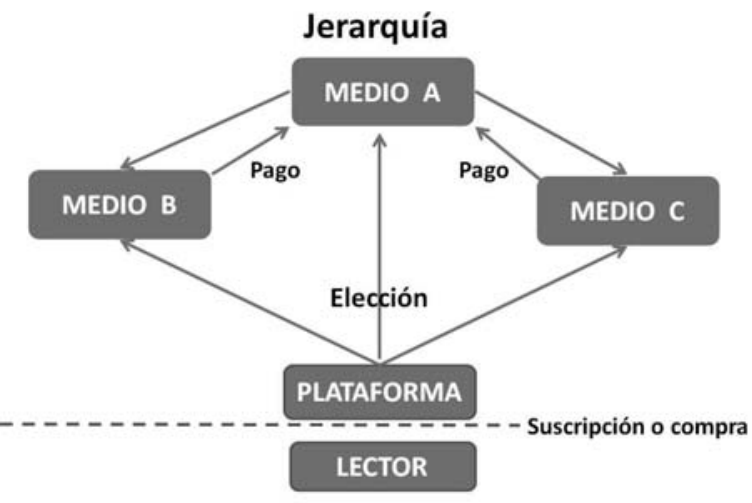

Representación de la alianza estratégica limitada. Elaboración propia en que mientras en las estrategias limitadas un medio de comunicación es el creador de la plataforma y le vende su tecnología al resto de medios, en las conjuntas, son varios medios de comunicación los que invierten en la creación de la plataforma.

La única alianza estratégica limitada de referencia en España es la plataforma Orbyt desarrollada por Unidad Editorial. La plataforma permite la subscripción o pago por uso a diarios integrados en diferentes grupos mediáticos pero vendidos a través de una única plataforma. El grupo creador (Unidad Editorial) posee los derechos de la plataforma y vende la tecnología a los grupos interesados (Grupo Zeta, Grupo Prensa

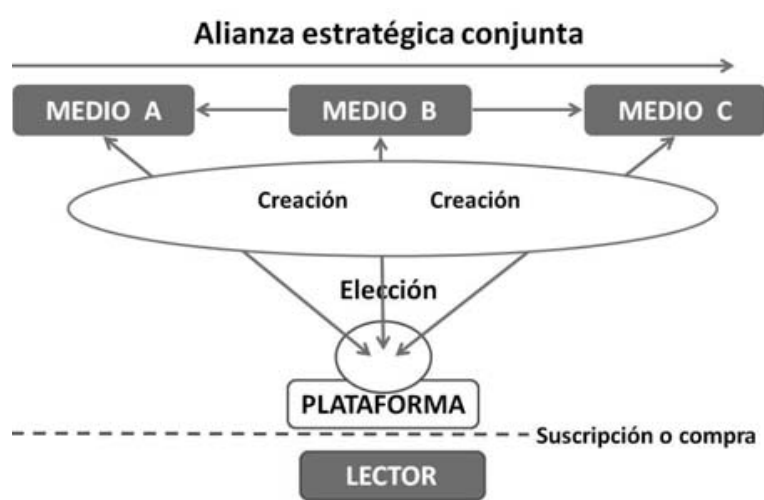

Representación de la alianza estratégica conjunta. Elaboración propia Ibérica, Hachette y Grupo Planeta). La alianza estratégica limitada [y conjunta] es un modelo de negocio que traduce el modelo de negocio tradicional a través de un quiosco virtual.

Las alianzas estratégicas conjuntas, no obstante, son acuerdos empresariales entre varias firmas con el objetivo de desarrollar una única plataforma de pago. No existe jerarquía y todas las firmas creadoras operan bajo los mis- 
mos costes de producción. Las firmas no creadoras que deseen integrarse en la plataforma deben firmar acuerdos empresariales con las firmas creadoras. Una de las alianzas estratégicas pioneras fue Ongo, la plataforma construida por Gannet Co. Inc., The New York times Co. y The Washington Post Co.

En España el lanzamiento de Kiosko y Más fue el único lanzamiento de una alianza estratégica conjunta. Los creadores principales son los grupos Prisa y Vocento y cuentan con la participación de otros como Zeta, Axel Springer, RBA, América Ibérica, Godó, etc. La iniciativa es la mayor alianza editorial en Europa en virtud a la participación en el proyecto de más de 13 grupos mediáticos.

\section{Alianzas estratégicas ajenas}

Las alianzas estratégicas ajenas son acuerdos empresariales, pero en lugar de ser entre medios de comunicación suelen ser entre un gestor de contenidos ajeno a la industria tradicional mediática y los medios de comunicación. El gestor de contenidos crea la plataforma y se encarga de su mantenimiento mientras los medios de comunicación que opten por este modelo le ceden una parte de sus ingresos.

Uno de los ejemplos pioneros y de mayor éxito es el modelo de subscripción de la App Store de Apple (Newsstand). La plataforma permite a los editores fijar el precio y el período de subscripción, mientras Apple procesa todos los pagos, reteniendo el $30 \%$ de los mismos. La principal controversia radica en la imposición que obliga a las editoras a ofrecer como mínimo las mismas ofertas en la App Store que fuera de ella,

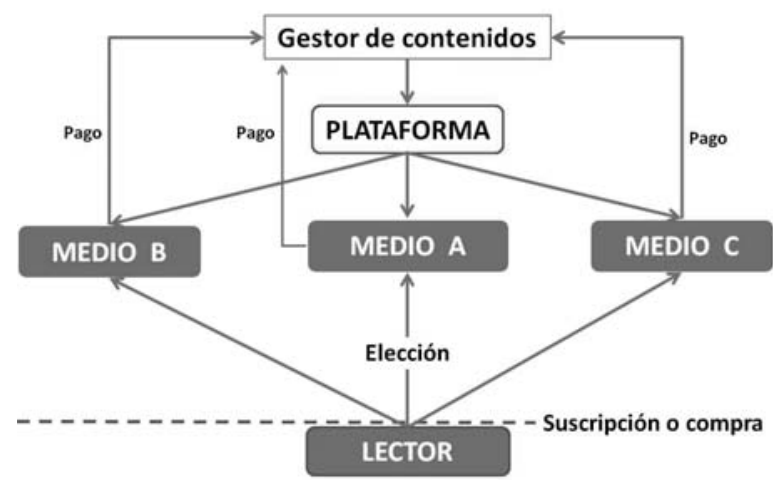

Representación de la alianza estratégica ajena. Elaboración propia prohibiendo además de forma explícita los enlaces para realizar esa subscripción fuera del acuerdo de comisiones con Apple (Véase, Apple, 2011).

\section{Conclusiones}

La carencia de un modelo de negocio paradigmático y extrapolable en la prensa digital ha provocado el inicio de una etapa de experimentación y ensayo de nuevas estrategias y modelos de negocio. La velocidad de Internet impide que grandes sectores e incluso, el académico, lleguen a asimilar con precisión el significado de los términos "estrategia" y "modelo de negocio" con la consiguiente confusión terminológica.

Bajo esta perspectiva, dividimos las estrategias de la prensa digital en estrategias propias, o en alianzas estrategias. Las estrategias propias pueden ser catalogadas como: gratuitas o de pago. Las estrategias propias gratuitas son las más abundantes, mientras las estrategias de pago pueden adoptar diferentes modalidades: cerradas (paywall) o mixtas (metered model o fremium). Las alianzas estratégicas no obstante, son (la 
mayoría de las veces) de pago y las clasificamos como propias o ajenas. Las propias pueden ser asimismo limitadas (cuando una plataforma es creadora por un medio o grupo de comunicación y vende su tecnología a otros grupos de comunicación, ej. Orbyt) o conjuntas (cuando varios grupos de comunicación crean una única plataforma, ej. Ongo y/o Kiosko y Más). Las alianzas estratégicas ajenas por otra parte son acuerdos empresariales entre un gestor de contenidos ajenos a la industria tradiconal mediática y grupos de comunicación (ej. Newsstand de Apple, Google One Pass, Livestand de Yahoo!, etc.).

\section{Referencias bibliográficas}

ALBORNOZ, Luís (2003): "La prensa digital en busca de modelos de negocio. Todo lo que el dinero puede comprar". Telos, $\mathrm{n}^{\circ}$ 57, pp. 131-133.

ALBORNOZ, Luís (2005): Los diarios online de información general: El caso de los grandes periódicos en español. Madrid, Universidad Complutense.

APPLE (2011): “Apple estrena subscripciones en la App Store": http://www.apple .com/es/pr/library/2011/02/15 Apple-Launches-Subscriptions-on-the-AppStore.html. [fecha de consulta: 10/02/2012]

BAKOS, Yannis y BRYNJOLFSSON, Erik (1996): "Bundling information goods: Pricing, profits and efficiency": http://people.stern.nyu.edu/bakos/big.pdf. [fecha de consulta: 29/01/2012]

BELL, Emily (2005): “End of the offline?". British Journalism Review, n ${ }^{\circ}$. London, Sage publications, pp. 41-45.

CAMPOS, Francisco (2010): "Las empresas de medios de comunicación revisan y amplían sus modelos de negocio". Razón y Palabra, n 74 .

CASADESUS, Ramón y RICART, Joan Enric (2009). "From Strategy to Business Models to Tactics". Harvard Business Review School, pp. 10-36.

CASERO-RIPOLLÉS, Andreu (2010): "Prensa en internet: nuevos modelos de negocio en el escenario de la convergencia". El profesional de la información, $\mathrm{n}^{\circ} 16$, pp. 595-601.

CASTELLS, Manuel (2000): La sociedad red. Madrid, Alianza Editorial.

CASTELLS, Manuel (2009): Comunicación y poder. Madrid, Alianza Editorial.

CHYI, Iris y SYLVIE, George (2001): "The medium is global, the content is not: The role of geography in online newspaper markets". Journal of Media Economics, $\mathrm{n}^{\circ}$ 4, pp.231-248.

COHEN, Heidi (2007): "What 'time on site' means for online media", en Clickz.com: http://www.clickz.com/clickz/column/1701460/what-time-site-means-onlinemedia. [fecha de consulta: 28/08/2010]

COMSCORE (2010): "UK newspapers online sites", en ComScore: $\mathrm{http}: / /$ www.comscore.com/. [fecha de consulta: 09/02/2012] 
CROBIE, Vin (2004): “Content or malcontent?", en Clitz.com: http://www.clickz .com/showPage.html?page=3298841. [fecha de consulta: 25/08/2009]

DRUCKER, Peter (1980): Managing in Turbulent Times. New York, HarperCollins

EDITORSWEBLOG (2011): "Newspapers Paywalls: When Metered Works, and when it doesn't", en EditorsWeblog: http://www.editorsweblog.org/newspaper/2011/06/newspaper_paywalls_when_metered_works_an.php. [fecha de consulta: 01/02/2012]

EISENBERG, Bryan (2002): "The ROI of free", en Clitz:com: http://www.clickz .com/clickz/column/1699775/the-roi-free. [fecha de consulta: 29/01/2012]

FAY, Scott y MACKIE-MASON, Jeffrey (2001): "Competition between firms that bundle information goods" http://deepblue.lib.umich.edu/bitstream/2027.42 /50428/1/bundle-8aug03.pdf. [fecha de consulta: 29/01/2012]

FILLOUX, Fredéríc (2011): "Analyzing the metered model" en Monday Note: http://www.mondaynote.com/2011/06/05/analyzing-the-metered-model/. [fecha de consulta: 27/01/2012]

GALIFORD, Miles (2010): "Why will people for content they could problably find for free on the Internet", en SubHub: http://www.subhub.com/articles/why-willpeople-pay-for-content-on-the-internet [fecha de consulta: 27/01/2012]

GALLARDO, Fernando (2011): "Nuevos modelos de negocio para la prensa". Telos, $\mathrm{n}^{\circ} 86$.

HUANG, Edgar (2007): "Use of rich media differs at newspaper, TV Web sites" Newspaper Research Journal, $\mathrm{n}^{\circ}$ 3, pp. 84-97.

HERBERT, Jack \& THURMAN, Neil (2007): "Paid content strategies for news websites". Journalism Practice, no 2, pp. 208-226.

KRÜGER, Corneilia; SWATMAN, Paula; and VAN DER BEEK, Kornelia (2003): "Business model formation within the online news market: The core + complement business model framework", en: 16th Bled Electronic Commerce Conference o eTransformation: https://domino.fov.uni-mb.si/proceedings.nsf/0/3a9bdcfcc 3ed0b5ec1256ea1002d0205/\$FILE/23Kruger.pdf [fecha de consulta: 08/03/2012]

JHONSON, Gerry (2001): Dirección Estratégica. Madrid, Prentice Hall.

KPMG (2010): "Anytime, anywhete. The rising demand of media on the movel" Londres.

LESSARD, Martin (2006): "Les seis cultures d'Internet", en ZeroSeconde: http://zeroseconde.blogspot.com/2006/09/les-6-cultures-dinternet.html [fecha de consulta: 05/09/2011].

LARRAÑAGA, Julio (2010): "Economía de los contenidos online: Periódicos online y e-books". Icono-14, n'2, pp. 1-37.

MAGRETTA, Joan (2002): "Why Business Models Matter". Harvard Business Review $\mathrm{n}^{\circ} 80 . \mathrm{pp} .86-92$. 
MENSING, Donica \& REJFECK, Jackie (2005): "Prospects for profit: The (un)evolving business model for online news", presentado en el International Symposium on Online Journalism: http://online.journalism.utexas.edu/2005/papers/mensing.pdf. [fecha de consulta: 08/03/2012]

MICÓ-SAN, Josep-Lluís y GONZÁLEZ-MOLINA, Sonia (2010): “Convergencia en la comunicación empresarial en las oficinas de prensa de tráfico y seguridad vial". El profesional de la información, $\mathrm{n}^{\circ} 19$. pp. 133-139.

MIGUEL DE BUSTOS, Juan Carlos (2000): "Industrias culturales, gratuidad y precios en Internet". Zer, $\mathrm{n}^{\circ} 9$.

MIGUEL DE BUSTOS, Juan Carlos y CASADO DEL RÍO, Miguel Ángel (2010): "Sobre pago y gratuidad". Telos, no 83.

MILLÁN, José Antonio (2001): "Del papel a la Red", en Jamillán: http://jamillan.com/papelred.htm. [fecha de consulta: 27/01/2012]

MINTZBERG, Henry (1994): The Rise and Fail of Strategic Planning. New York, Free Press.

NESBITT, Mary y LAVINE, John (2004): Reaching new readers: Revolution, not evolution. Evanston, IL, Northwestern University Readership Institute.

O'REGAN, Rob (2011): "Metered Model gains Traction as digital pay option" en EmediaVitals: http://emediavitals.com/content/metered-model-gains-traction-digital-pay-option. [fecha de consulta: 01/02/2012]

PAIDCONTENT (2011a): "Interview: Digital's second age begins now, FT CEO says", en PaidContent: http://paidcontent.co.uk/article/419-interview-digitals-second-age-begins-now-ft-ceo-says/ [fecha de consulta: 10/02/2012]

PAIDCONTENT (2011b): "How FT a NYT Aim to Make Paywalls Pay", en PaidContent: http://paidcontent.org/article/419-how-the-ft-and-nyt-aim-to-make-paywalls-pay/. [fecha de consulta: 10/02/2012]

PAIDCONTENT (2011c): "NYT co sees Drop in Earnings But 20\% Rise in Digital Subs" en PaidContent: http://paidcontent.org/article/419-nyt-co-see-drop-in-earnings-but-20-rise-in-digital-subs/. [Consultado: 10/02/2012]

PENG, Foo, et al. (1999): "Trends in online newspapers: A look at the US web", en Newspaper Research Journal: http://www.questia.com/googleScholar.qst ?docId=5002320220. [fecha de consulta: 08/03/2012]

PFANNER, Eric (2007): "Norwegian newspaper publisher finds the secret to profiting online", en New York Times: http://www.nytimes.com/2007/02/18/business/worldbusiness/18iht-papers.4633531.html?pagewanted=all. $[$ fecha de consulta: 25/10/2009]

PEW RESEARCH CENTER (2011): "The Tablet Revolution and What it Means for the Future of News", en Pew Research: http://pewresearch.org/pubs/2119/tabletnews [fecha de consulta: 19/01/2012] 
PICARD, Robert (1988): "Measures of concentration in the daily newspaper industry". Journal of Media Economics, n ${ }^{\circ}$ 1, pp. 61-74.

PICARD, Robert (1999): "Cash cows or entrecote: Publishing companies and disruptive technologies". Trends in communication, $\mathrm{n}^{\mathrm{o}}$ 11, pp. 127-136.

PICARD, Robert (2009): "Why Journalist deserve low pay", en Reuters Institute for the Studi of Journalism: http://tinyurl.com/7anwwcu. [fecha de consulta: 25/09/2011]

ROCHET, Jean-Charles \& TIROLE, Jean (2004): Two-Side Markets: An Overview, en IDEI-CEPR: http://faculty.haas.berkeley.edu/hermalin/rochet tirole.pdf. [fecha de consulta: 25/02/2012]

RODRÍGUEZ-MARTÍNEZ, Ruth; CODINA, Lluís; y PEDRAZA-JIMÉNEZ Rafael (2010): "Cibermedios y web 2.0: modelo de análisis y resultados de aplicación". El profesional de la información, $\mathrm{n}^{\circ} 19$, pp. 35-44.

ROJO-VILLADA, Pedro Antonio (2008): Modelos de negocio y consumo de prensa en el contexto digital. Murcia, Universidad de Murcia, Servicio de Publicaciones.

SALINGER, Michael (1995): "A graphical analysis of bundling” The Journal of Business, $\mathrm{n}^{\circ} 68$, pp.85-98.

SCHMALENSEE, Richard (1984): "Gaussian demand and commodity bundling" The Journal of Business, n57, pp.211-230.

SHAPIRO, Carl \& VARIANT, Hal (1998): Information Rules: A Strategic Guide to the Network Economy. New York, Prentice Hall.

SHAFER, Scott, et al. (2005): The Power of Business Models. Business Horizons, $\mathrm{n}^{\circ}$ 48. pp. 199-207.

STRUPP, Joe (2007): "Paid sites seem less likely as TimesSelect exits", en Editor \& Publisher: http://www.journalism.org/node/7627. [fecha de consulta: 05/06/2011]

SYLVIE, George (2008): "Developing an online newspaper business model: long distance meets the long tail". Online Journalism. pp. 1-39.

THE GUARDIAN (2010): “Times Lost Almost 90\% of online Readership", en The Guardian: http://www.guardian.co.uk/media/2010/jul/20/times-paywall-readership [fecha de consulta: 08/03/2012]

VARELA, Juan (2011): "Periodismo mutante". Telos, no 86

VARIANT, Hal (1995): "Pricing information goods" en Proceedings of Scholarship in the New Information Environment Symposium: http://hubcap.clemson.edu/ sauerr/classes/425/cases/varina_price-info-goods.pdf [fecha de consulta: 08/01/2012]

ZERDICK, Axel, et al. (1999): "Die Internet-Ökonomie. Strategien für die digitale Wirtschaft" [The Internet economy: Strategies for the digital economy] (3rd ed.). Heidelberg, Germany, Springer. 